\title{
Planet Consumption and Stellar Metallicity Enhancements
}

\author{
Eric Sandquist ${ }^{1}$, Ronald E. Taam ${ }^{1}$, D. N. C. Lin $^{2}$, Andreas Burkert ${ }^{3}$ \\ ${ }^{1}$ Department of Physics \& Astronomy, Northwestern University, Evanston, IL 60208 \\ erics@apollo.astro.nwu.edu, taam@ossenu.astro.nwu.edu \\ ${ }^{2} \mathrm{UCO} /$ Lick Observatory, Board of Studies in Astronomy and Astrophysics, University of \\ California, Santa Cruz, CA 95064 \\ lin@ucolick.org \\ ${ }^{3}$ Max Planck Institut for Astronomy, Koenigstuhl 17, D-69117 Heidelberg, Germany \\ burkert@mpia-hd.mpg.de
}

\begin{abstract}
The evolution of a giant planet within the stellar envelope of a main-sequence star is investigated as a possible mechanism for enhancing the stellar metallicities of the parent stars of extrasolar planetary systems. Three-dimensional hydrodynamical simulations of a planet subject to impacting stellar matter indicate that the envelope of a Jupiter-like giant planet can be completely stripped in the outer stellar convection zone of a $1 M_{\odot}$ star. In contrast, Jupiter-like and less massive Saturn-like giant planets are able to survive through the base of the convection zone of a $1.22 M_{\odot}$ star. Although strongly dependent on details of planetary interior models, partial or total dissolution of giant planets can result in significant enhancements in the metallicity of host stars with masses in the range $1.0 M_{\odot} \lesssim M \lesssim 1.3 M_{\odot}$. The implications of these results with regard to planetary orbital migration are briefly discussed.
\end{abstract}

Subject headings: stars: abundances, stars: planetary systems

\section{INTRODUCTION}

With the detection of planets orbiting around nearby solar-type stars (Mayor \& Queloz 1995; Butler \& Marcy 1996; Marcy \& Butler 1998) there has been renewed interest in the origin and orbital evolution of planetary systems. Particularly noteworthy about a large fraction of the discoveries was the small separations (less than $0.2 \mathrm{AU}$ ) at which the Jupiter-sized planets orbited about their parent stars. Based on our current understanding of the formation of the giant planets, it is likely that they formed at large distances from their parent stars (on the order of a few AU) where temperatures in the protoplanetary disk were sufficiently low that refractory material could condense. Subsequently the giant planets must have undergone significant inward orbital migration either due to tidal interaction with the remnant disk and star (Lin, Bodenheimer, \& 
Richardson 1996; Trilling et al. 1998; Murray et al. 1998) or to gravitational interactions between the planets themselves (Rasio \& Ford 1996) during the remaining lifetime of the system.

Investigations of the chemical composition of the stellar companions in these systems (Gonzalez 1997, 1998a) indicate that the metallicity of the stellar photospheres is enhanced above the solar value, which is itself at the high end of the metallicity distribution of nearby G-dwarfs (Favata, Micela, \& Sciortino 1997). This suggests that either metal-rich environments are more conducive to the formation of these types of planetary systems, or that orbital migration has led to the accretion of planetary bodies into the stellar envelope (Lin 1997; Gonzalez 1997, 1998ab; Jeffrey, Bailey, \& Chambers 1997; Laughlin \& Adams 1997) since gas giants in the solar system appear to have average metal contents of $\mathrm{Z} \gtrsim 0.1$ (Gudkova \& Zharkov 1990). In this interpretation, the metallicity enhancement is restricted to their outermost envelope layers and is not indicative of the star as a whole.

In this Letter we explore the accretion hypothesis and report on the three-dimensional hydrodynamical evolution of a planet moving through the envelope of its parent star. The goals of this investigation are the determination of the amount of mass removed from the planet due to mass stripping and shock heating, and the consequent metallicity enhancement expected for the star. Provided that the planetary envelope is at least partially removed and/or the planetary core is melted and vaporized within the outer stellar convection zone, the metallicity of the stellar envelope can be enhanced.

\section{FORMULATION}

In this study it is assumed that the planet is in a circular orbit about its parent (main sequence) star and, due to computational resource limitations, is already partially immersed in the outermost regions of the stellar envelope. The orbital evolution immediately prior to this phase is governed by the action of gravitational tidal torques with the star (Lin 1997). Low-mass main sequence stars brake their rotation on a timescale of approximately $10^{8} \mathrm{yr}$ (Skumanich 1972) once the protoplanetary disk has been dissipated, allowing the star to exert drag on the planet. This spiral-in phase and the possible mass loss from the planet associated with tidal effects has not been followed, and it is beyond the scope of the present investigation. (This scenario would occur later than the pre-main sequence scenario considered by Laughlin \& Adams 1997). The structure of the host star was calculated from a stellar evolutionary code developed by Eggleton $(1971,1972)$ and updated by Pols et al. (1995). The star in the first simulation had a mass of $1 M_{\odot}$ and an age of $4.65 \times 10^{9}$ years, with a convection zone containing $0.02 M_{\odot}$ and extending over the outer $28 \%$ of its radius. A $1.22 M_{\odot}$ model at an age of $10^{9}$ years (having an outer convection zone of $0.003 M_{\odot}$ and a fractional radial extent of $18 \%$ ) was used in the remaining two simulations. For the planet, we use polytropic models with an index $n$ equal to 1.0 or 1.5 , which are good approximations to the density structure of Jupiter and Saturn, respectively (Hubbard 1984). 
The three-dimensional hydrodynamical evolution of the planet within the stellar envelope is calculated using a nested grid technique in a code developed by Burkert \& Bodenheimer (1993) as described in Sandquist et al. (1998). The computational domain is composed of two grids of $64 \times 64 \times 64$ zones having total physical sizes of $5.2 \times 10^{10} \mathrm{~cm}$ and $1.3 \times 10^{10} \mathrm{~cm}$ respectively. The subgrid was positioned over the center of the planet, and subsequently we followed the planet's center of mass reference frame by imprinting the orbital motion of the planet with respect to the stellar envelope on the velocity field of the inflowing matter. The subgrid was placed off-center in the main grid in order to allow us to follow more of the disturbed flow.

The orbit of the planet decays as the result of momentum transfer from the stellar to the planetary envelope. Gravitational focusing and drag is unimportant in the present circumstance since the accretion radius is more than a factor of 50 times smaller than the planet's radius for the conditions under consideration here (viz., orbital velocity of $4.4 \times 10^{7} \mathrm{~cm} \mathrm{~s}^{-1}$ and a planetary mass and radius comparable to that of Jupiter $\sim 0.001 M_{\odot}$ and $\left.\sim 0.1 R_{\odot}\right)$. The aerodynamic drag force acting on the planet's surface was taken into account by computing the planet's center-of-mass velocity relative to the grid at each step, and correcting the planet's orbital motion accordingly. The instantaneous mass of the planet was computed as the sum of zones in the subgrid for which the speed was less than $20 \%$ of the orbital motion of the planet. The planet was recentered if it moved more than 0.3 of a grid zone, thus ensuring adequate spatial resolution.

A number of simplifying approximations have been introduced in the problem to facilitate computation. For example, we have implicitly assumed that the planet is always encountering an inflowing density distribution corresponding to the undisturbed stellar envelope. This approximation should be adequate during the planet's main mass loss phase since the timescale for orbital evolution is shorter than the orbital period during this phase. On the other hand, this approximation is suspect in the early evolution where the planet skims the surface of the star, making orbits at approximately the same radius. However, very little mass is expected to be lost during this stage due to the low densities in the stellar atmosphere, so the total mass loss from the planet is less likely to be significantly affected. Perhaps a more serious approximation is the lack of treatment of the transfer of orbital angular momentum to spin angular momentum of the stellar material. Angular momentum exchange arises from tidal interaction as well as mass transfer. This results in an overestimate of the relative velocity and an underestimate of the timescale for the evolution of the planet within the stellar envelope. The effect on mass loss from the planet should not be large since the region in which the planet has significant gravitational influence on the incoming stellar matter is much smaller than its own radius, and the planet's interaction with disturbed stellar matter is not expected to be significant. Finally, the gas in the stellar envelope and in the planet is treated as a perfect gas, and the compositional differences between the planetary material and the stellar envelope are neglected in the simulations. This latter approximation should not significantly affect the overall results, however, since the energy required to dissociate and ionize the gas within the planetary envelope is much less than the energy input associated with the impact of the stellar material. 


\section{NUMERICAL RESULTS}

Three numerical simulations of a planet within the envelope of a main sequence star have been performed. In the first sequence we examined the impact of the stellar envelope in the $1 M_{\odot}$ star on a Jupiter-like giant planet. The initial structure of the planet was described by an $n=1$ polytrope with mass and radius equal to the mass, $M_{J}$, and radius, $R_{J}$ of Jupiter. The planet was initially placed in orbit at a radius of $0.98 R_{\odot}$.

As illustrated in Fig. 1 the distance of the planet from the center of the star rapidly decreased from $0.98 R_{\odot}$ to $0.78 R_{\odot}$ in about $17000 \mathrm{~s}$. The mass of the planet only decreased by $11 \%$ on the first orbit (about $9900 \mathrm{~s}$ ). As the planet plunged deeper into the stellar envelope (for depths greater than $0.05 R_{\odot}$ from the stellar surface - see Fig. 1), the mass loss from the planet increased dramatically. In this simulation, the planet lost $90 \%$ of its mass once it has passed through $80 \%$ of the convection zone's radial extent. The planet was significantly distorted from its initial spherical distribution, most rapidly on the side that was deeper in the stellar envelope. The leading edge was also significantly compressed. The effect of shock heating is clearly evident in Fig. 2 where the entropy distribution in the flow is illustrated for two snapshots. The regions of high entropy in the flow identify regions that were shock heated. At the end of the simulation, the stronger shock was contributing to quicker mass loss from the planet.

To determine the sensitivity of the results to the assumed mass of the parent star, the $1 M_{\odot}$ star was replaced with a $1.22 M_{\odot}$ star in the second and third simulations. Because the orbital decay timescale is longer in this case, we started the planet in orbit at 0.96R. By the time the planet reached the base of the convection zone, it had lost a smaller amount of mass $\left(\sim 0.3 M_{J}\right)$ due to the lower densities in the convection zone. Although the orbital decay timescale in this sequence is comparable to the orbital period of the planet during the main mass loss phase (so that the planet is likely to encounter stellar material disturbed by its previous passage), the planet is still unlikely to dissolve in the convection zone. This is a consequence of the feedback between the amount of momentum incident on the planet and the planet's rate of orbital decay: a higher (lower) momentum flux results in a faster (slower) decay. As a result, a large change in the mass loss from the planet is less likely.

In the final simulation, we sent a Saturn-like planet through the envelope of a $1.22 M_{\odot}$ star. Here, the initial interior structure of the planet is described by a polytropic index of 1.5 and the planet is characterized by a mass and radius of $0.3 M_{J}$ and $0.81 R_{J}$ respectively. Figure $1 \mathrm{~b}$ illustrates that the planet's orbit decayed more quickly than did Jupiter's. Because Saturn has a more centrally-concentrated mass distribution than Jupiter, the steeper density gradients were better able to inhibit the inward propagation of shock waves, resulting in momentum transfer to the planet as a whole, rather than mass loss. In agreement with this idea, our Saturn-like planet lost less mass $\left(0.09 M_{J}\right)$ than our Jupiter-like planet did $\left(0.17 M_{J}\right)$ after both had swept through the same amount of mass in the stellar envelope $\left(0.23 M_{J}\right)$. 


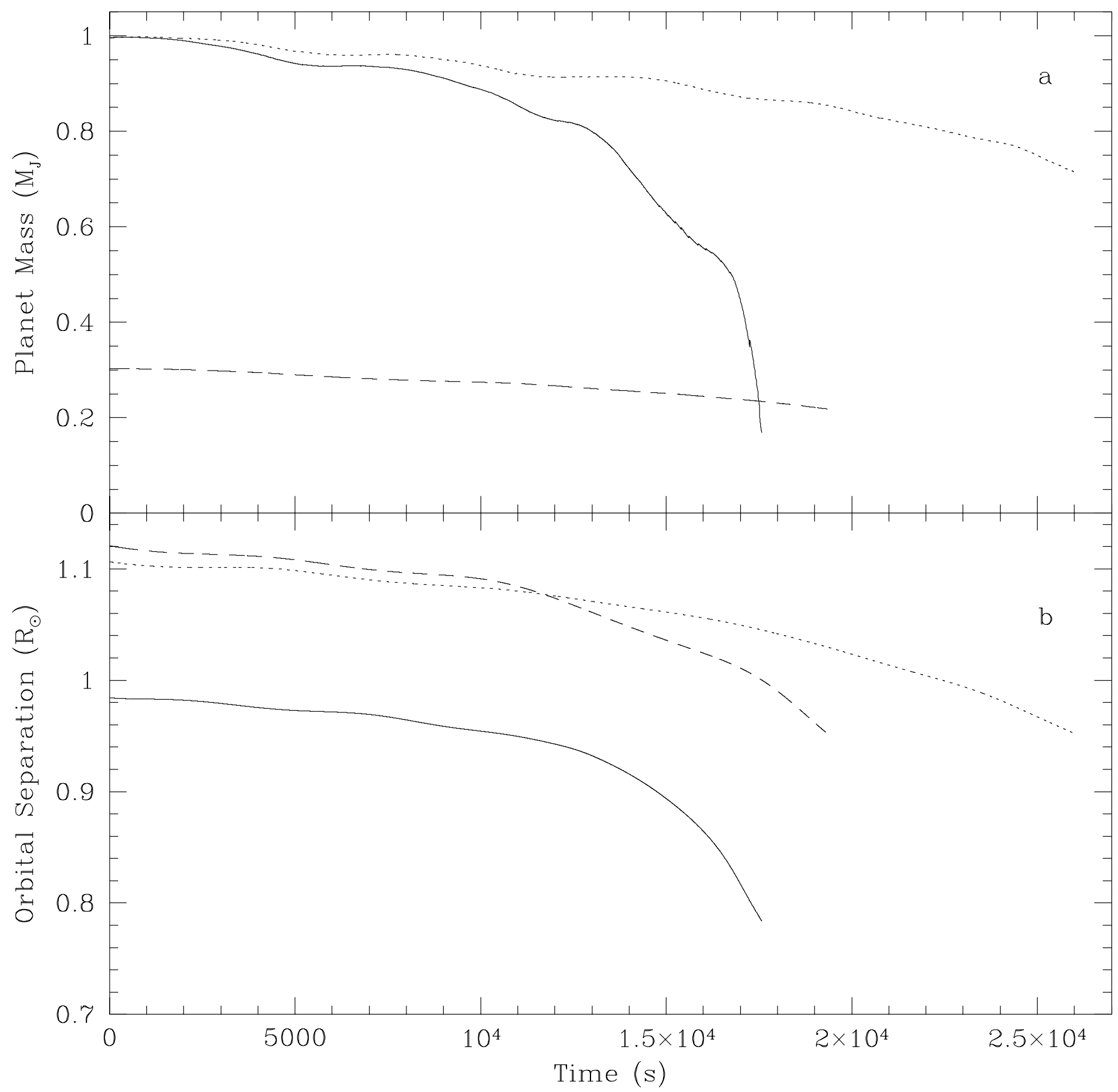

Fig. 1. - a) The temporal variation of the planet mass for the Jupiter - $1 M_{\odot}$ star simulation ( solid line), the Jupiter - $1.22 M_{\odot}$ star simulation (dotted line), and the Saturn - $1.22 M_{\odot}$ star simulation (dashed line). b) The temporal variation of the orbital separation for the three simulations. 

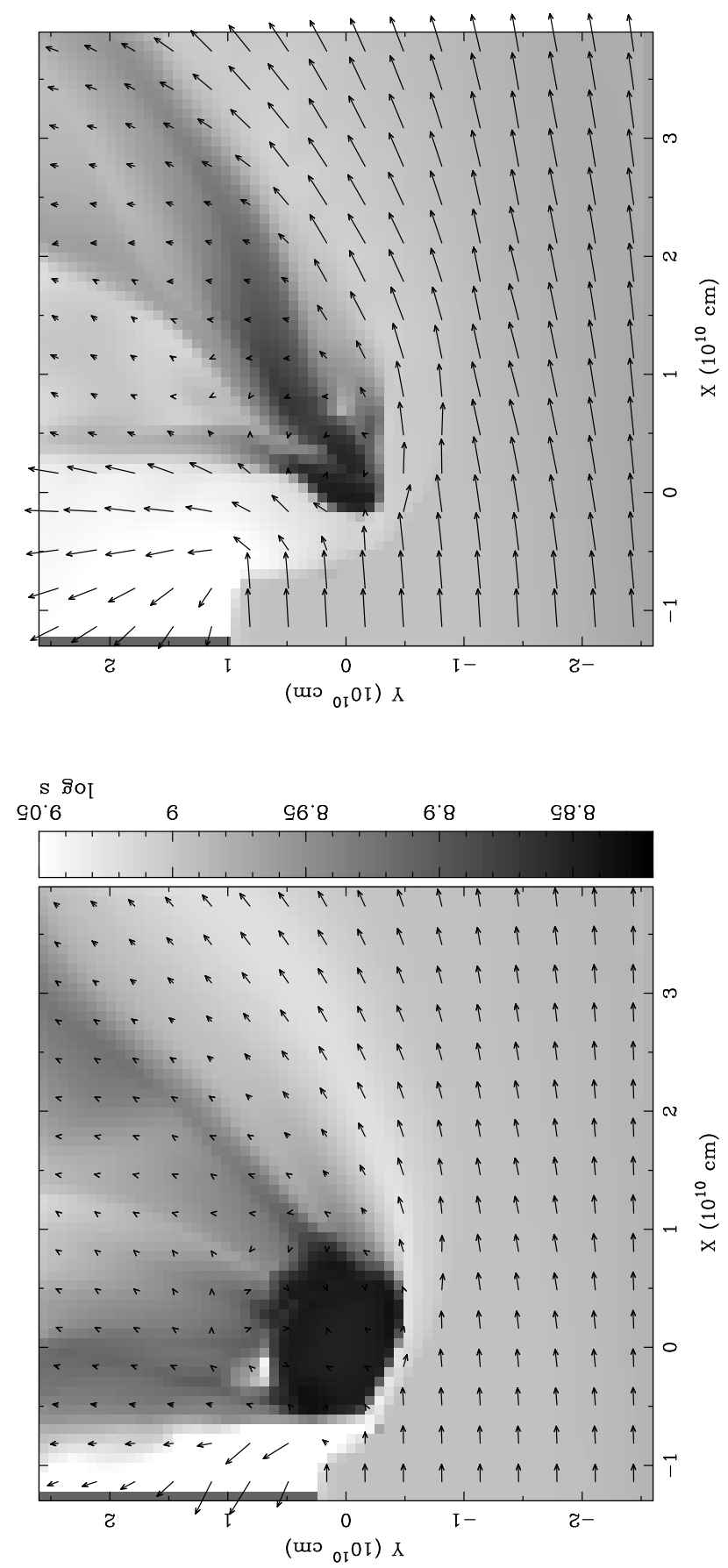

Fig. 2.- The entropy distribution and velocity field in the meridional plane of the Jupiter-like planet in the envelope of a $1 M_{\odot}$ star at times a) $6740 \mathrm{~s}$, and b) $15999 \mathrm{~s}$. The $x$-and $y$-axes correspond to the orbital direction and the radial direction respectively. The entropy of the original surface of the planet was $\log s=8.91$. The velocity vectors are scaled to the maximum in-plane speed. The maximum speeds for the panels are: a) $985 \mathrm{~km} \mathrm{~s}^{-1}(6740 \mathrm{~s})$, and b) $468 \mathrm{~km} \mathrm{~s}^{-1}$ (15999 $\mathrm{s})$. 


\section{DISCUSSION}

Before discussing the potential metallicity enhancements that could be observed as a result of star-planet interactions like the ones we have simulated here, we must first summarize current beliefs regarding the chemical composition and interior structure of the gas giants in the solar system. Recent models (Guillot, Gautier, \& Hubbard 1997) indicate that the total amount of elements heavier than helium in Jupiter is between 11 and $45 M_{\oplus}$, with the preferred equation of state implying a mass of less than $33 M_{\oplus}$. The interior distribution of metals is not well-constrained - there are no observable differences if the metals are distributed uniformly though the metallic hydrogen region, or are condensed into a dense core. Though the core-instability model for giant planet formation requires a core mass of about $15 M_{\oplus}$ to initiate rapid gas accretion for Jupiter (Podolak et al. 1993, Pollack et al. 1996), the mass of the core can decrease during the formation phase due to heating by accreting material. Rock/ice cores of masses between 2 and $12 M_{\oplus}$ appear to be necessary with Guillot et al.'s preferred equation of state. Models of extrasolar planets (Burrows et al. 1997) have hinted at the presence of radiative zones in planets like Jupiter and Saturn. This can be relevant if significant heavy-element enrichment of the planet's envelope resulted from the impact of rock/ice planetesimals after it had accreted the majority of its gas.

From three-dimensional hydrodynamical simulations of the evolution of a Jupiter-like planet in the convective envelope of a solar-mass star, we found that the planetary envelope can be completely stripped away. If the planet has a solid core, it is also likely to be melted and vaporized in the convection zone since the rate of heating associated with the impacting matter $\left(\sim \pi R^{2} \rho v^{3} \sim 10^{40} \mathrm{ergs} \mathrm{s}^{-1}\right)$ is sufficient to dissolve the core on a short timescale $(\sim 10-100 \mathrm{~s})$. Thus, the dissolution of a single Jupiter-like planet could lead to a heavy element enrichment of between 3 and $26 \%$ in the envelope of a star of solar mass and metallicity. Greater metallicity enhancements in solar-type stars could be achieved if a few Jupiter-like planets or large numbers of planetesimals were accreted. The likelihood of these kinds of accretion events depends on a number of unknown factors like the mass function for planets, the lifetime of the protoplanetary disk, and the orbital history of the planets and residual debris. In determining the metallicity enhancements, we have also assumed that there is no elemental diffusion or mixing across the radiative-convective boundary. Efficient mixing across this boundary would result in some depletion of light elements like lithium and beryllium (although the planet itself would introduce a fresh supply), and would modify the internal angular momentum distribution of the star.

The numerical simulation for a $1.22 M_{\odot}$ star reveals that a Jupiter-like planet loses about $30 \%$ of its envelope mass in the stellar convection zone. The heavy-element enrichment of the star could reach above $50 \%$ if Jupiter's heavy elements are distributed uniformly through its envelope. However, this is still subject to the considerable uncertainty in the planet composition models -

no enrichment would be observed if the heavy elements were concentrated in Jupiter's core. In the simulation using a Saturn-like planet, the metallicity enhancement would be about $14 \%$ at most, and the planet model uncertainties are greater. Models of Saturn imply a total metal mass of up to $20 M_{\oplus}$ including a rock-ice core of approximately $7 M_{\oplus}$ (Gudkova \& Zharkov 1990). 
The envelope structure of the host star is clearly an important factor in the metallicity enrichment by planets since less massive convection zones could magnify the enhancements. However, main-sequence stars more massive than the Sun have thinner and less dense convection zones, and so they are not as effective in stripping the planetary envelope. Meridional circulation, induced by the angular velocity gradients resulting from the deposition of orbital angular momentum in the stellar envelope, is not likely to be suppressed in the radiative envelope of stars more massive than about $1.4 M_{\odot}$. Hence, the metals are expected to be well mixed in a region of the stellar envelope which is affected by rotational instabilities. In a separate paper, we will examine the extent of metal enhancement in these more massive stars. The deeper and higher density convection zones in stars less massive than the Sun would be more effective in stripping, but their greater mass more effectively dilutes the added metals. Since the convection zone mass rapidly increases with decreasing stellar mass, stars significantly less massive than the Sun are unlikely to show metallicity enhancements even if they consume several planets. Based on the calculations presented here, if a solar-mass star consumed one Jupiter-like planet, its metallicity would only be enhanced by at most 0.1 dex (if the star initially had solar metallicity). Thus, we expect that metallicity enhancements can only be observed in stars ranging from about $1.0-1.3 M_{\odot}$.

Gonzalez (1998a) has measured abundances and estimated masses for most of the stars believed to have planetary systems. He finds that the four "51 Peg-like" systems (51 Peg, v And, $\tau$ Boo, $\left.\rho^{1} \mathrm{Cnc}\right)$ have a mean abundance $[\mathrm{Fe} / \mathrm{H}]=+0.25$, and two $\left(\tau\right.$ Boo and $\left.\rho^{1} \mathrm{Cnc}\right)$ appear to belong to the small group (about 8 members) of known "super metal-rich" stars (Taylor 1996). The stars with planet candidates were selected as being solar-type stars, so the star sample does not tell us in a general sense whether stars with planets fall in the range where we would predict that metallicity enhancements are possible. However, of the four, $v$ And and $\tau$ Boo have mass estimates of approximately $1.3 M_{\odot}, 51 \mathrm{Peg}$ has about $1.1 M_{\odot}$, and $\rho^{1} \mathrm{Cnc}$ may be as low at $0.7 M_{\odot}$ (Gonzalez 1998a, Ng \& Bertelli 1998).

The orbital angular momentum deposited in the stellar convection zone may influence the orbital migration of the remaining planets in the system. For example, if the surface layers of the central star are spun up as a result of the angular momentum deposited in the envelope, then the remaining planets within several stellar radii may spiral outwards if the stellar spin frequency is greater than the orbital frequency of the planet. The angular momentum deposited in the stellar surface layers may also facilitate dynamo action, enhancing the magnetic field of the central star. This can lead to enhanced magnetic braking of the stellar surface, thereby spinning down the star. Spectroscopic observations of stars with extrasolar planet candidates show mixed evidence for higher spin rates, as $\tau$ Boo and $v$ And have $v \sin i$ of about $10 \mathrm{~km} \mathrm{~s}^{-1}$ (Gonzalez 1997), and all others have $v \sin i$ of just a few $\mathrm{km} \mathrm{s}^{-1}$ (Gonzalez 1998a).

Residual planetesimals, comets, and short-period terrestrial planets may also contaminate the host stars with heavy elements. In one scenario for the origin of short-period planets, Murray et al. (1998) suggest that a giant planet can induce eccentricity growth among residual 
planetesimals through resonant interactions. Subsequent close encounters cause most of the affected planetesimals to be ejected outwards while the planet migrates inward. A Jupiter-mass planet could only migrate a large distance inward if there was a substantial population of planetesimals within its orbit. At the distance of a few stellar radii, some fraction of the close encounters would send the planetesimals toward their host stars to be dissolved in the outermost layers of the stellar envelope. The total mass of planetesimals needed to explain all of the heavy elements contained in the convective envelope of solar-type stars (a few tens of Earth masses) is considerably smaller than what is required to induce a Jupiter-mass to undergo any significant orbital decay. Unless most of the acquired heavy elements are able to diffuse into the radiative interior, the planetesimal-scattering scenario for orbital migration would require more than $90 \%$ of the close encounters to result in the outward ejection of planetesimals.

We wish to acknowledge discussions with Prof. Peter Bodenheimer. This research was supported by NASA under grant NAG5-7515 and by NSF grants AST-9415423, AST-9618548, and AST-9727875. 


\section{REFERENCES}

Burkert, A. \& Bodenheimer, P. 1993, MNRAS, 264, 798

Burrows, A., Marley, M., Hubbard, W. B., Lunine, J. I., Guillot, T., Saumon, D., Freedman, R., Sudarsky, D., \& Sharp, C. 1997, ApJ, 491, 856

Butler, R. P. \& Marcy, G. W. 1996, ApJ, 464, L153

Eggleton, P. P. 1971, MNRAS, 151, 351

Eggleton, P. P. 1972, MNRAS, 156, 361

Favata, F., Micela, G., \& Sciortino, S. 1997, A\&A, 323, 809

Gonzalez, G. 1997, MNRAS, 285, 403

Gonzalez, G. 1998a, A\&A, 334, 221

Gonzalez, G. 1998b, in Brown Dwarfs and Extrasolar Planets, ed. R. Rebolo, (Provo: ASP Conference Proceedings), in press

Gudkova, T. V. \& Zharkov, V. N. 1990, Sov. Ast. Lett., 16, 401

Guillot, T., Gautier, D., \& Hubbard, W. B. 1997, Icarus, 130, 534

Hubbard, W. B. 1984, Planetary Interiors, (New York: Van Nostrand-Reinhold)

Jeffery, C. S., Bailey, M. E. \& Chambers, J. E. 1997, Observatory, 117, 224

Laughlin, G. \& Adams, F. 1997, ApJ, 491, 51L

Lin, D. N. C. 1997, in Accretion Phenomena and Related Outflows, IAU Colloquium 163, ed. L. Ferrario \& D. Wickramsinghe, in press

Lin, D. N. C., Bodenheimer, P. \& Richardson, D. C. 1996, Nature, 380, 606

Marcy, G. W. \& Butler, R. P. 1998, Ann. Rev. Astr. Ap., in press

Marcy, G. W., Butler, R. P., Williams, E., Bildsten, L, Graham, J. R., Ghez, A. M. \& Jernigan, J. G. 1997, ApJ, 481, 926

Mayor, M. \& Queloz, D. 1995, Nature, 378, 355

Murray, N., Hansen, B., Holman, M., \& Tremaine, S. 1998, Science, 279, 69

Ng, Y. K. \& Bertelli, G. 1998, A\&A, 329, 943

Podolak, M., Hubbard, W. B., \& Pollack, J. B. 1993, in Protostars and Planets III, eds. E. H. Levy \& J. I. Lunine, (Tucson: University of Arizona Press), 1109

Pollack, J.B., Hubickyi, O., Bodenheimer, P., Lissauer, J.J., Podolak, M., \& Greenzweig, Y. 1996, Icarus, 124,62 .

Pols, O. R., Tout, C., Eggleton, P. P. \& Han, Z. 1995, MNRAS, 274, 964

Rasio, F. \& Ford, E. B. 1996, Science, 274, 954

Sandquist, E., Taam, R. E., Chen, X., Bodenheimer, P. \& Burkert, A. 1998, ApJ, in press 
Skumanich, A. 1972, ApJ, 171, 565

Taylor, B. J. 1996, ApJS, 102, 105

Trilling, D. E., Benz, W., Guillot, T., Lunine, J. I., Hubbard, W. B. \& Burrows, A. 1998, ApJ, 500,428

Yorke, H. W., Bodenheimer, P. \& Laughlin, G. 1993, ApJ, 411, 274 